\title{
Análisis del rendimiento de la mano de obra en la construcción del rubro de enlucido liso en la ciudad de Cuenca
}

\begin{abstract}
(1) (1) (-)
\end{abstract}
Analysis of the performance of labor in construction in the area of smooth plastering in the city of Cuenca

Juan Diego Cabrera Valdez. ${ }^{1}$ \& Jorge Fernando Toledo Toledo. ${ }^{2}$

Recibido: 03-08-2021 / Revisado: 15-08-2021 /Aceptado: 31-08-2021/ Publicado: 05-11-2021

\begin{abstract}
DOI: $\underline{\text { https://doi.org/10.33262/concienciadigital.v4i4.1.1921 }}$

Objective. The present research work aims to obtain the different factors of affectation of the performance of labor for the workers that make up a crew for the field of smooth plastering in the construction of houses in the city of Cuenca. Methodology. The research was carried out using a qualitative exploratory approach where the starting data were obtained through a survey that was applied to civil engineers contractors, who participate in the processes of SERCOP (National Public Procurement Service). The methodology proposed by Antonio Cano and Gustavo Duque in 2000 for obtaining factors affecting labor performance was replicated. Results. This research focused on the analysis of the factors of climate, equipment, supervision, labor aspects and worker conditions. Conclusion. Finally, we identified which of these variables generate a positive impact by increasing the performance of the labor force.
\end{abstract}

Keywords: performance, labor, crew, smooth render, factors of affectation.

\footnotetext{
1 Universidad Católica de Cuenca, Maestría en Construcciones con Mención en la Administración de Construcción Sustentable, Azuay, Ecuador, juandiego.cabrera1@est.ucacue.edu.ec, ORCID https://orcid.org/0000-0003-0367-4951.

${ }^{2}$ Universidad Católica de Cuenca, Facultad de Arquitectura. Cuenca, Ecuador, jftoledot@ucacue.edu.ec. ORCID https://orcid.org/0000-0003-0007-7510.
} 


\section{Resumen}

Objetivo. El presente trabajo de investigación tiene como objetivo obtener los diferentes factores de afectación del rendimiento de mano de obra para los obreros que conforman una cuadrilla para el rubro de enlucido liso en la construcción de viviendas en la ciudad de Cuenca. Metodología. La investigación se realizó utilizando un enfoque cualitativo de tipo exploratorio donde los datos de partida se obtuvieron mediante una encuesta que se aplicó a ingenieros civiles contratistas, que participan en los procesos del Servicio Nacional de Compras Públicas (SERCOP). Se replicó la metodología propuesta por Antonio Cano y Gustavo Duque en el año 2000 para la obtención de factores de afectación del rendimiento de mano de obra. Resultados. Esta investigación se enfocó en el análisis de los factores de clima, equipamiento, supervisión, aspectos laborales y condiciones propias de trabajador. Conclusión. Finalmente se identificaron cuáles de estas variables generan un impacto positivo al incrementar el rendimiento de la mano de obra.

Palabras claves: rendimiento, mano de obra, cuadrilla, enlucido liso, factores de afectación.

\section{Introducción}

El sector de la construcción es considerado una parte importante en el desarrollo de cada país, no solo por la contribución al crecimiento económico, sino también por la generación de empleo. En Ecuador, éste representó el 8.17\% del PIB y generó 6.1\% del total de empleos del año 2019 (Lucero, 2020). Un proyecto de construcción implica el empleo de una gran cantidad de recursos, por lo que una correcta elaboración del presupuesto sirve como una herramienta de control de obra; de allí la necesidad de elaborarlo de manera detallada y estimada (Janampa, 2021). El análisis de precios unitarios es parte importante en la obtención de este presupuesto. El mismo está integrado por la suma de los materiales requeridos para el rubro, la relación de equipo y herramienta, y la mano de obra necesaria para la ejecución de dicha tarea (Lascano, 2015).

En ecuador, el costo de la mano de obra representa de un $28 \%$ a un $40 \%$ del gasto total en los costos de construcción y por tanto su rendimiento y consumo son temas que, conociéndolos, permiten al constructor ser competitivo dentro de un mercado en el cual cada vez es más difícil participar (Calle, 2013). Ya que no existen bases de datos que permitan obtener rendimientos nace la problemática de no poseer información clara y puntual para obtener rendimientos reales y con esto planear adecuadamente un proyecto. Esto obliga a cuestionar la confiabilidad de los análisis de precios unitarios utilizados por los constructores, instituciones públicas y privadas (Arboleda, 2014; Mejía y Hernández, 2007).

Varios autores han estudiado el tema de construcción, productividad, presupuestos, rendimientos de mano de obra y factores que influyen en ella. Serpell (1986) concluyó que la productividad en la ejecución de los proyectos de construcción se ve afectada por varios factores como: administración de la obra, entorno en el que se desarrolla la obra, tipo y método de trabajo y personal del proyecto. Cano y Duque (2000). señalaron nuevos 
factores de afectación para el cálculo del rendimiento de la mano de obra exponiéndolos en siete categorías. Y, además desarrollaron una metodología para evaluar dichos factores en forma normalizada asignándoles un porcentaje de eficiencia a cada uno de ellos, donde la evaluación global la obtuvieron tomando la media aritmética de los porcentajes asignados para los siete grupos. Así se puede encontrar la eficiencia que afecta a los rendimientos de cualquier actividad de construcción.

Los factores de afectación señalados por Cano y Duque (2000) son: 1) Economía general, donde los factores dependen del estado económico del área o país donde se desarrolla el proyecto. El rendimiento es inverso al estado del país, es decir cuando la economía del país es buena, la eficiencia del rendimiento baja y viceversa. 2) Aspectos Laborales, aquí se consideran las condiciones laborales en las que se realiza el proyecto, como experiencia, disponibilidad, contratación y salarios. 3) Clima, ya que el estado del tiempo debe ser considerado, especialmente factores como tiempo, temperatura, condiciones de suelo y disponibilidad de cubierta. 4) Actividad, aquí se tienen en cuenta las condiciones específicas del trabajo y depende de su alcance, condiciones del sitio, suministro de materiales y mecanización de operaciones. 5) Equipamiento, donde la disponibilidad de equipo apropiado, su estado, mantenimiento y reparación afectan el rendimiento esperable del proyecto. 6) Supervisión, donde factores como dirección, seguimiento, instrucciones e idoneidad del maestro son analizados. 7) Trabajador, los aspectos personales se deben considerar ya que estos afectan el desempeño. Se toman en cuenta factores como estado de ánimo, situación familiar, habilidad, conocimientos y condición física.

Para la presente investigación se aplicó la metodología planteada por Cano y Duque (2000), para analizar el rubro de enlucido de una vivienda. Según el INEN (2015), una vivienda es definida como una edificación con luces que no excedan $5.0 \mathrm{~m}$ y que no supere dos niveles en altura en ninguna de sus fachadas. Además, se denomina enlucido al revestimiento continuo que constituye la capa de terminación aplicada sobre la superficie con el fin de tapar la mampostería, vigas o columnas que soportan la misma. De esa manera quedan lisas, facilitando la aplicación posterior del empastado y pintura $u$ otros tipos de acabado. Existen varios tipos de enlucidos: paleteado grueso, liso, escobillado fino, champeado, tarrajeado y escobillado grueso (Cchorahua, 2016). El alcance de esta investigación se enfocará en el enlucido liso.

Se han realizado algunas investigaciones para encontrar un rendimiento promedio de enlucidos lisos en nuestro medio aplicando la metodología propuesta por Cano y Duque (2000). Es así que Del Valle y Jativa (2018) calcularon un rendimiento promedio de 0.253 h/m2 para tres obras ubicadas en la ciudad de Guayaquil. Lascano (2015) calculó un rendimiento de $0.52 \mathrm{~h} / \mathrm{m} 2$ para un edificio en la ciudad de Guayaquil, por otro lado y para condiciones similares, Brenes (2014) calculó un rendimiento de $0.517 \mathrm{~h} / \mathrm{m} 2$ en edificaciones prefabricadas en Costa Rica, y Dávila (2020) calculó un rendimiento de $0.10 \mathrm{~h} / \mathrm{m} 2$ en Huánuco, Perú. El rendimiento final encontrado por estos autores varía significativamente, por lo cual se hace necesario analizar la causa de tan alta dispersión. 
El método propuesto permite identificar los factores causantes y cuantificar su grado de influencia en dicha dispersión.

Por todo lo mencionado anteriormente el presente trabajo de investigación tiene como objetivo obtener los diferentes factores de afectación del rendimiento de mano de obra para los obreros que conforman una cuadrilla, es decir un maestro principal y un oficial en el rubro de enlucido liso en la construcción de viviendas en la ciudad de Cuenca.

\section{Metodología}

Para la obtención de los factores de afectación del rendimiento de mano de obra para los obreros que conforman una cuadrilla en el rubro de enlucido liso en la construcción de viviendas en la ciudad de Cuenca se utilizó la metodología planteada por Cano y Duque (2000). El presente proyecto fue una investigación cualitativa de tipo exploratorio. Para la población en estudio se usó la técnica de elección por juicio, debido a que la selección de la muestra fue basada en la credibilidad del investigador. Una vez establecida la población, se realizó un muestreo aleatorio simple (Hernández et al., 2015).

La obtención de datos se efectuó a través de encuestas y su instrumento, el cuestionario. Según García (2003), la encuesta es un procedimiento que sirve para la obtención y registro de datos, su versatilidad permite utilizarlo como instrumento de investigación y de evaluación de personas, procesos y programas de formación. El cuestionario de esta encuesta constó de 12 preguntas, las cuales se estructuraron de manera que se respondan cualitativamente, con los siguientes índices de evaluación: muy baja, baja, normal, muy buena y excelente. La encuesta se realizó en línea mediante la herramienta de Google encuestas.

Una vez realizada las encuestas, los resultados de cada pregunta fueron categorizados en tablas de frecuencias. Luego, se dio un valor cuantitativo de eficiencia (de 0 a $100 \%$ ) a cada uno de estos parámetros según lo descrito en la metodología propuesta por Cano y Duque (2000). Por último, estas eficiencias esperadas se comparan entre ellas para ver en qué condiciones de trabajo se espera obtener mejores rendimientos y de qué manera estos afectan al rendimiento total esperado.

\section{Población y muestra}

Debido a que se analizó el rubro de enlucido liso, la elección de la población de estudio se basó en ingenieros civiles que ofertan y participan en el SERCOP en la ciudad de Cuenca, ya que los procesos emitidos por esta entidad poseen este rubro. En el SERCOP existen alrededor de 1300 ingenieros civiles inscritos, que participan en los procesos de contratación pública donde consta el rubro del enlucido (SERCOP, 2021). Cabe recalcar que para este estudio se tomó en cuenta los ingenieros que participan ya sean personas naturales o jurídicas.

Ya que la investigación es de tipo cualitativa con población finita, Dicovskiy (2008) recomienda emplear un muestreo aleatorio simple (Ecuación 1) para la elección de la muestra. Esta ecuación define el tamaño de la muestra (n), tomando en cuenta factores 
como el tamaño de la población $(\mathrm{N})$, la variancia de una distribución binomial $(\mathrm{p}=\mathrm{q}=$ $0.5)$, y un valor $\mathrm{D}=\mathrm{B}^{2} / 4$ obtenido mediante un error de estimación prefijado $(\mathrm{B}=0.09$ ). La muestra calculada para esta investigación fue de 113 personas, por lo que se realizaron 115 encuestas.

$$
n=\frac{N * p * q}{(N-1) * D+p * q} \quad \text { Ecuación (1) }
$$

Análisis de factores de afectación

Cano y Duque (2000). en su investigación "Rendimientos y consumos de mano de obra", analizan los llamados "factores de afectación". Estos factores que normalmente no se tienen en cuenta al momento de tomar los datos de rendimiento en obra son los causantes de una alta dispersión en los resultados obtenidos por diferentes autores. Indican que un rendimiento está normalizado cuando muestra el desempeño de la mano de obra de manera natural, es decir libre de factores que la afecten. Además, señalan que este rendimiento normal está en un punto cercano al $70 \%$ dentro de un rango de 0 a $100 \%$, estableciendo una escala de eficiencia en los rendimientos, resumida en la tabla 1.

Tabla 1

\begin{tabular}{lc} 
Escala de eficiencia en los rendimientos de mano de & \multicolumn{2}{c}{ Eficiencia en la productividad } & Rango \\
\hline Muy baja & $10 \%-40 \%$ \\
Baja & $41 \%-60 \%$ \\
Normal (promedio) & $61 \%-80 \%$ \\
Muy buena & $81 \%-90 \%$ \\
Excelente & $91 \%-100 \%$ \\
\hline
\end{tabular}

Fuente: Page (1997), tomado de Estimator's general construction man hour manual.

El objetivo de su trabajo fue desarrollar una metodología que permita medir la cantidad de recurso humano consumido al elaborar una cantidad unitaria de cualquier actividad de construcción. Esta metodología permite captar todos los datos requeridos para la completa evaluación de los rendimientos y el análisis de los factores que las afectan, dividida en dos partes, la primera clasifica y entiende los factores de afección, dado como resultado la clasificación de éstos en siete grupos: economía general, aspectos laborales, clima actividad, equipamiento, supervisión, y trabajador. La segunda parte de la metodología propuesta desarrolló una evaluación matemática de los factores antes estudiados para poder comparar el rendimiento medido en obra con el rendimiento normalizado.

Para el desarrollo de esta investigación, no se incluyó el factor de economía general pues éste produce una reacción en cadena con los otros grupos de factores y la actualidad económica del país se ha visto afectada por la pandemia del coronavirus (Lucero, 2020). El factor de obra tampoco fue incluido, pues este trabajo se desarrolla exclusivamente para el enlucido de viviendas. Es por esto, que en el análisis presentado en esta investigación se abordaron solamente las variables de clima, equipamiento, supervisión, 
aspectos laborables; y trabajador ya que se consideraron medibles, cuantificables y relevantes para el análisis del rendimiento en el rubro de enlucido liso.

La metodología planteada por Cano y Duque (2000), evalúa cada una de las categorías en las que se clasificaron los factores de afección, asignándoles un porcentaje de eficiencia donde la evaluación global se obtiene tomando la media aritmética de los porcentajes obtenidos para los siete grupos. Los autores definieron rangos de calificación para cada uno de los factores dados, ya que ciertos factores afectan en mayor medida el desempeño de la mano de obra. En la tabla 2 se presentan estos rangos y sus equivalencias respectivas para una escala de calificación similar a la obtenida en las encuestas realizadas: muy baja (1), baja (2), normal (3), muy buena (4) y excelente (5).

\section{Tabla 2}

Rangos de eficiencia y equivalencias de los factores de afectación del rendimiento de mano de obra

\begin{tabular}{lccccccc}
\hline \multicolumn{1}{c}{ Grupo } & Rango (\%) & $\mathbf{0}$ & $\mathbf{1}$ & $\mathbf{2}$ & $\mathbf{3}$ & $\mathbf{4}$ & $\mathbf{5}$ \\
\hline Economía general & 50 a 75 & 50 & 55 & 60 & 65 & 70 & 75 \\
Clima & 40 a 75 & 40 & 47 & 54 & 61 & 68 & 75 \\
Obra & 40 a 80 & 40 & 48 & 56 & 64 & 72 & 80 \\
Equipamiento & 55 a 75 & 55 & 59 & 63 & 67 & 71 & 75 \\
Supervisión & 50 a 75 & 50 & 55 & 60 & 65 & 70 & 75 \\
Laborales & 40 a 80 & 40 & 48 & 56 & 64 & 72 & 80 \\
Trabajador & 60 a 75 & 60 & 63 & 66 & 69 & 72 & 75 \\
\hline
\end{tabular}

Fuente: Cano y Duque (2000), tomado de Rendimientos y consumos de mano de obra.

El costo unitario de mano de obra obtenido al multiplicar el rendimiento normalizado por el costo hora de cada uno de los obreros que conforman la cuadrilla, debe ser afectado por la calificación global del rendimiento o productividad esperable calculada con el método descrito. Una calificación de eficiencia global obtenida del 65\%, comparado con el rendimiento normalizado de eficiencia alrededor de $70 \%$, indica que el costo unitario de mano de obra debe ser incrementado en un 5\%. De manera similar si la eficiencia global obtenida es de $75 \%$, el costo unitario deber ser minorado en este $5 \%$.

\section{Resultados}

Una vez realizadas las encuestas, se tabularon y procesaron los datos obtenidos. Una vez aplicada la metodología de análisis propuesta se obtuvieron las tablas resumen de cada una de las variables analizadas, las mismas muestran la tendencia obtenida por los contratistas en relación al rendimiento de mano de obra en el rubro de enlucido.

\section{Variable Clima-Temperatura:}

Se analizó la eficiencia esperada en clima lluvioso, soleado y ventoso. La tabla 3 muestra que el rendimiento esperado en un clima lluvioso o soleado es el mismo, esto se puede deber a que es una actividad realizada en el interior y bajo cubierta, por lo que el clima 
no afecta el rendimiento esperado. El valor de eficiencia obtenido para estas variables fue de $63 \%$ lo que representa un rango normal de trabajo. Por otra parte, para una condición de clima ventoso la eficiencia calculada es de $69 \%$, clasificado como un rendimiento muy bueno según los encuestados. Al trabajar en condiciones de clima ventoso, la eficiencia aumenta en un $6 \%$ a la esperada en otras condiciones.

Tabla 3

Eficiencia real calculada para diferentes condiciones de clima

\begin{tabular}{lcccc}
\hline Eficiencia del & Valor de afectación & \multicolumn{3}{c}{ Condición estudiada } \\
\cline { 3 - 5 } rendimiento & $(\%)$ & 0 & 0 & 0 \\
\hline Muy baja (1) & 47 & 19 & 22 & 3 \\
Baja (2) & 54 & 53 & 50 & 9 \\
Normal (3) & 61 & 37 & 34 & 72 \\
Muy buena (4) & 68 & 6 & 9 & 31 \\
Excelente (5) & 75 & 62.83 & 62.89 & 68.97 \\
\hline
\end{tabular}

Eficiencia real calculada (\%): $\quad 62.83 \quad 62.89 \quad 68.97$

Fuente: Elaboración propia

\section{Variable equipamiento}

La tabla 4 muestra la eficiencia esperada cuando los obreros cuentan con equipo de protección personal (EPP). Esta eficiencia es de 71\%, la misma que se encuentra en el rango de eficiencia muy buena, lo que indica que esta variable influye de manera positiva en el rendimiento real esperado. Si bien el uso de EPP es obligatorio durante las actividades de construcción, esto no siempre se cumple. Por esta razón las preguntas de la encuesta fueron realizadas a contratistas que han podido palpar la realidad de nuestro medio y pueden emitir un criterio del impacto que esto posee en el rendimiento.

\section{Tabla 4}

Eficiencia real calculada para diferentes condiciones de equipamiento

\begin{tabular}{lcc}
\hline $\begin{array}{c}\text { Eficiencia del } \\
\text { rendimiento }\end{array}$ & $\begin{array}{c}\text { Valor de } \\
\text { afectación (\%) }\end{array}$ & $\begin{array}{c}\text { Condición estudiada } \\
\text { EPP }\end{array}$ \\
\hline Muy baja (1) & 59 & 0 \\
Baja (2) & 63 & 19 \\
Normal (3) & 67 & 9 \\
Muy buena (4) & 71 & 53 \\
Excelente (5) & 75 & 34 \\
\hline \multicolumn{2}{c}{ Eficiencia real calculada (\%): } \\
\hline
\end{tabular}

Fuente: Elaboración propia

\section{Variable Supervisión}

En esta variable se analizaron tres condiciones: tener responsabilidades definidas, materiales y pagos a tiempo. Las eficiencias se muestran en la Tabla 5, aquí se puede ver que el rendimiento real calculado para todas las condiciones planteadas se encuentra en 
un rango de eficiencia muy bueno, siendo el hecho de tener los materiales en obra a tiempo el factor que más aumenta la eficiencia. Las variables analizadas evitan problemas en la coordinación debido a una mala organización y mala planificación (Mora Valverde, 2012) por lo cual una alta eficiencia era esperada como resultado al realizar este análisis.

Tabla 5

Eficiencia real calculada para diferentes condiciones de supervisión

\begin{tabular}{lccccc}
\hline \multirow{2}{*}{$\begin{array}{c}\text { Eficiencia del } \\
\text { rendimiento }\end{array}$} & $\begin{array}{c}\text { Valor de } \\
\text { afectación (\%) }\end{array}$ & $\begin{array}{c}\text { Materiales } \\
\text { a tiempo }\end{array}$ & $\begin{array}{c}\text { Responsabilidades } \\
\text { definidas }\end{array}$ & $\begin{array}{c}\text { Pagos a } \\
\text { tiempo }\end{array}$ \\
\cline { 1 - 4 } Muy baja (1) & 47 & 0 & 0 & 0 \\
Baja (2) & 54 & 0 & 0 & 0 \\
Normal (3) & 61 & 25 & 34 & 31 \\
Muy buena (4) & 68 & 50 & 72 & 68 \\
Excelente (5) & 75 & 40 & 9 & 16 \\
\hline \multicolumn{2}{c}{ Efficiencia real calculada (\%): } & 68.91 & 66.48 & 67.09 \\
\hline
\end{tabular}

Fuente: Elaboración propia

\section{Variable Aspectos Laborales}

Para la evaluación de esta variable se realizaron dos análisis, el primero presentado en la tabla 6 se realizó comparando el tipo de contrato que el contratista aplicaría al momento de contratar a los obreros. Se puede apreciar que la eficiencia real calculada es mayor cuando se subcontrata la obra, siendo esta eficiencia un $9 \%$ mayor que la obtenida con la condición de pago semanal. Además, se observa que al pagar algún anticipo esta eficiencia sube aún más, llegando al rango de muy buena.

Tabla 6

Eficiencia real calculada para diferentes condiciones de contrato

\begin{tabular}{lcccc}
\hline \multirow{2}{*}{$\begin{array}{c}\text { Eficiencia del } \\
\text { rendimiento }\end{array}$} & \multirow{2}{*}{$\begin{array}{c}\text { Valor de } \\
\text { afectación (\%) }\end{array}$} & \multicolumn{3}{c}{ Condición estudiada } \\
\cline { 1 - 3 } \cline { 4 - 5 } Muy baja (1) & 48 & 0 & 6 & 3 \\
Baja (2) & 56 & 3 & 25 & 6 \\
Normal (3) & 64 & 9 & 62 & 31 \\
Muy buena (4) & 72 & 87 & 19 & 50 \\
Excelente (5) & 80 & 16 & 3 & 25 \\
\hline \multicolumn{2}{c}{ Eficiencia real calculada (\%): } & 72.07 & 63.17 & 70.12 \\
\hline
\end{tabular}

Fuente: Elaboración propia

El segundo análisis consideró variables como la afiliación al IESS, incentivos laborales y la existencia de un correcto ambiente laboral, como se muestra en la tabla 7. La afiliación al IESS muestra una baja en la eficiencia del rendimiento hasta un $61 \%$, un valor menor al esperado de eficiencia normal y por tanto afectando de forma negativa en el cálculo del rendimiento. Se puede identificar el descontento de los trabajadores hacia la afiliación obligatoria, pues si bien ésta posee varios beneficios para los empleados, solamente toman 
en cuenta el porcentaje del sueldo reducido debido a esta. Por otra parte, la asignación de actividades con recompensas por labor cumplida, favorece el mejoramiento de la productividad de la mano de obra y un ambiente de trabajo cordial entre compañeros, personal obrero y jefes suman un ambiente de trabajo en las que se garantiza que el factor humano tendrá un mayor desempeño.

Tabla 7

Eficiencia real calculada para diferentes condiciones de aspectos laborales

\begin{tabular}{|c|c|c|c|c|}
\hline \multirow{2}{*}{$\begin{array}{l}\text { Eficiencia del } \\
\text { rendimiento }\end{array}$} & \multirow{2}{*}{$\begin{array}{c}\text { Valor de } \\
\text { afectación } \\
(\%)\end{array}$} & \multicolumn{3}{|c|}{ Condición estudiada } \\
\hline & & $\begin{array}{c}\text { Afiliación al } \\
\text { IESS }\end{array}$ & $\begin{array}{c}\text { Buen } \\
\text { ambiente }\end{array}$ & Incentivos \\
\hline Muy baja (1) & 48 & 31 & 0 & 0 \\
\hline Baja (2) & 56 & 3 & 0 & 0 \\
\hline Normal (3) & 64 & 65 & 47 & 25 \\
\hline Muy buena (4) & 72 & 13 & 37 & 47 \\
\hline Excelente (5) & 80 & 3 & 31 & 43 \\
\hline \multicolumn{2}{|c|}{ Eficiencia real calculada $(\%)$ : } & 60.8 & 70.89 & 73.25 \\
\hline
\end{tabular}

Fuente: Elaboración propia

\section{Variable trabajador}

Para esta variable se consideró parámetros como: experiencia, contextura física, salud y edad. Con el análisis de estos parámetros se pudo determinar el nivel de rendimiento esperado por los ingenieros contratistas, mismo que se muestra en la tabla 8. Los contratistas no consideran que la eficiencia se vea afectada por condiciones como la contextura, salud o edad. Por otro lado, la experiencia si se considera un parámetro importante para aumentar la eficiencia, así se puede notar al ver la eficiencia esperada de $73 \%$, un valor similar a la impuesta en el rango de muy buena.

Tabla 8

Eficiencia real calculada para diferentes condiciones propias del trabajador

\begin{tabular}{lccccc}
\hline \multirow{2}{*}{$\begin{array}{c}\text { Eficiencia del } \\
\text { rendimiento }\end{array}$} & $\begin{array}{c}\text { Valor de } \\
\text { afectación (\%) }\end{array}$ & Experiencia & $\begin{array}{c}\text { Contextura } \\
\text { física }\end{array}$ & Salud & Edad \\
\hline Muy baja (1) & 63 & 0 & 0 & 0 & 0 \\
Baja (2) & 66 & 4 & 33 & 30 & 48 \\
Normal (3) & 69 & 15 & 41 & 41 & 34 \\
Muy buena (4) & 72 & 37 & 22 & 33 & 22 \\
Excelente (5) & 75 & 59 & 19 & 11 & 11 \\
\hline \multicolumn{2}{c}{ Eficiencia real calculada (\%): } & 72.94 & 69.70 & 69.65 & 68.90 \\
\hline
\end{tabular}

Fuente: Elaboración propia 


\section{Conclusiones}

- En los resultados presentados se resume el cálculo de los factores de afectación del rendimiento de mano de obra para los obreros que conforman una cuadrilla en el rubro de enlucido liso en la construcción de viviendas en la ciudad de Cuenca, con lo que se pude concluir que se cumplió el objetivo planteado para esta investigación.

- Las variables de equipamiento, supervisión y aspectos laborales son factores que influyen positivamente en el rendimiento obtenido y debido a que la mayoría de los parámetros que se incluyen en estas variables pueden ser controlados con una correcta planificación por parte del contratista, un aumento en el rendimiento de mano de obra es factible y depende en su mayoría del ingeniero a quien se le adjudique la obra.

- En el caso de la variable de clima, no existe una variación negativa en el cálculo de eficiencia debido a que, en su mayoría, las obras de enlucido se pueden realizar en el interior y bajo cubierta, más bien ciertas condiciones como el clima ventoso influyen positivamente en el rendimiento. Es importante notar que esta variable no puede ser controlada ni mejorada.

- El factor de afectación para el caso de las condiciones propias del trabajador tampoco muestra una variación significativa en los parámetros estudiados. Siendo la experiencia el único factor que los ingenieros contratistas señalan como realmente importante al tomar en cuenta esta variable.

- El factor de afectación global se calcula aplicando la media aritmética de los factores obtenidos para cada una de las variables estudiadas. En este trabajo, no se pudo determinar este factor total de afectación pues no existen datos tomados en obra ya que durante la elaboración de esta investigación la mayoría de obras estuvieron paradas debido a la pandemia del coronavirus, lo que limitó la obtención de esta información. Se recomienda para futuras investigaciones realizar un levantamiento de datos en campo y con ello obtener los parámetros necesarios para aplicar la metodología completa para el cálculo de rendimientos reales.

\section{Referencias bibliográficas}

Arboleda López, S. A. (2014). Análisis de productividad, rendimientos y consumo de mano de obra en procesos constructivos, elemento fundamental en fase de planeación. Universidad nacional de Colombia.

Brenes Serrano, J. O. (2014). Análisis de rendimientos y productividad de mano de obra para la empresa La Puerta del Sol Equipo Constructor S.A. Instituto Tecnológico de Costa Rica.

Calle Castro, C. J. (2013). Análisis de los rendimientos de mano de obra, equipo y materiales en edificaciones de hasta tres plantas en la cuidad de Azogues. Universidad de Cuenca. 
Cano, A., \& Duque, G. (2000). Rendimientos y Consumos de la Mano de Obra (p. 43). Sena - Camacol.

Cchorahua Chirinos, E. (2016). Estudio del rendimiento y productividad de la mano de obra en las partidas de asentado del muro de ladrillo, enlucido de cielo raso con yeso y tarrajeo de muros en la construcción del condominio residencial torre del sol. Universidad andina del cusco.

Dávila Falcón, R. (2020). Rendimiento del personal obrero en las partidas de muros y tabiques de albañilería, revoques y enlucidos y contrazocalos y su comparación con las normas CAPECO en la obra: Universidad nacional "Hermilio Valdizán."

Del Valle Wong, J. O., \& Jativa Cruz, L. G. (2018). Guía práctica para comparar los rendimientos y uso de los quipos, mano de obra, materiales en los análisis de precios unitarios de los rubros de movimiento de tierra, estructura y mampostería de una edificación en la contratación pública. Universidad Laica Vicente Rocafuerte de Guayaquil.

Dicovskiy, L. M. (2008). Estadística básica. In Universidad Nacional de Ingeniería (Vol. $1)$.

García, T. (2003). El cuestionario como instrumento de investigación/evaluación. 28. http://www.univsantana.com/sociologia/El_Cuestionario.pdf

Hernández Sampieri, R., Fernández Collado, C., \& Baptista Lucio, P. (2015). Metodología de la investigación (5ta edición). Mc Graw Hill.

INEN. (2015).Cpe Inen-Nec-Se-Vivienda Cap10, 113

Janampa Palomino, G. E. (2021). Análisis del rendimiento de mano de obra en las partidas tarrajeo de muros interiores y cielorraso, y su influencia en los costos reales de ejecución, en la construcción del Colegio Integrado Puerto Yurinaki Perené. Universidad Continental.

Lascano Iñiguez, M. A. (2015). Rendimiento de mano de obra de los principales rubros: comprobación real en el sitio de obra. Universidad católica Santiago de Guayaquil.

Lucero, C. (2020). La construcción, un pilar de la economía debilitado por la pandemia. Gestión Digital.

Mejía Aguilar, G., \& Hernández, T. (2007). Seguimiento de la Productividad en obra: técnicas de medición de rendimientos de mano de obra. 2, 45-59.

Page, J. (1997). Estimators general construcción man-hour manual (Segunda ed). Gulf Publishing Company.

SERCOP. (2021). Servicio Nacional de Contratación Pública. https://www.compraspublicas.gob.ec/ProcesoContratacion/compras/ 
Serpell, A. (1986). Productividad en la construcción. Revista Ingeniería de Construcción, $1,53-59$.

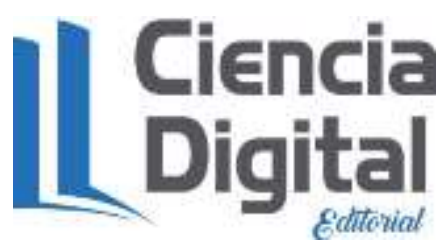




\section{PARA CITAR EL ARTÍCULO INDEXADO.}

Cabrera Valdez, J. D., \& Toledo Toledo, J. F. (2021). Análisis del rendimiento de la mano de obra en la construcción del rubro de enlucido liso en la ciudad de Cuenca. ConcienciaDigital, 4(4.1), 6-18. https://doi.org/10.33262/concienciadigital.v4i4.1.1921

\section{Ciencia Ligital \\ Edthorial}

El artículo que se publica es de exclusiva responsabilidad de los autores y no necesariamente reflejan el pensamiento de la Revista Conciencia Digital.

El artículo queda en propiedad de la revista y, por tanto, su publicación parcial y/o total en otro medio tiene que ser autorizado por el director de la Revista Conciencia Digital.

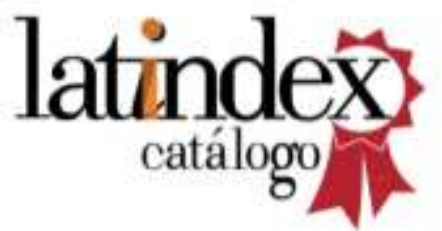

